\title{
Patients' Perception Towards the Preservation of Natural Teeth For Life
}

Jaafar N, Jallaludin RL, Razak IA, Esa R. Patients' Perception Towards the Preservation of Natural Teeth for Life. Annals Dent. Univ. Malaya 1995;2:37-40.

\begin{abstract}
The aim of the article is to report on the perception of utilizers of government dental services towards the preservation of natural teeth for life and relate it to their past utilization pattern and the associated demographic factors. All patients aged 15 years and over attending ten randomly selected government dental clinics in Selangor, Wilayah Persekutuan and Negri Sembilan during the study period of thirty nonconsecutive outpatients days were interviewed and examined clinically. Five-hundred and fifty subjects were included in the survey. It was found that the majority of respondents $(63 \%)$ have a.rather pessimistic perception of their ability to preserve natural teeth for life and most have a very poor past utilization behaviour ( $90 \%$ ). The most pessimistic and worst utilization behaviour was reported by the Malay ethnic group, the least formally educated and the lowest income group $(p<0.01)$. It is postulated that one of the main reason for this trend among Malay respondents could be due to their lower educational and income status, rather than cultural influences. Further research into the influence of culture on the utilization pattern of the Malays is therefore recommended.
\end{abstract}

Key Words: Oral health services research, Behwoioural science.

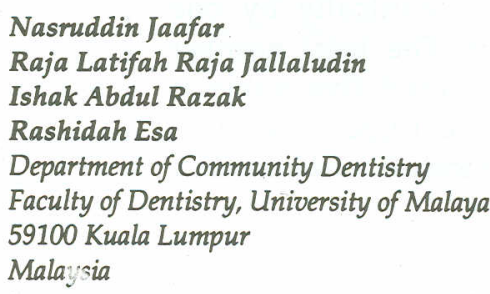

\section{INTRODUCTION}

The natural dentition is indeed intended to and should be able to last a lifetime (1). However for some people a pessimistic attitude towards retention of their natural teeth persist, coloured by their past disease and treatment experience. The rate at which natural teeth are lost is influenced by many factors including the attitudes of dentists, patients and the availability of dental services. It is ironic that the presence of dentists may actually speed up the rate of tooth loss as compared to populations where there are less dentists (1).

Data from the International Collaborative study in 1978 showed that one of the most important factors influencing tooth loss was consumers' perceived attitude towards restorations and dentures (2). It was evident that the availability of dental services could not prevent a very high rate of tooth loss and edentulousness by age 35-44 years in all the systems studied. This occured despite having favourable dentist:population ratios (3). Premature tooth loss was attributed mainly to consumers' attitude towards preference for extractions and making complete dentures, rather than subjecting themselves to an endless cycle of tooth restoration and repair (4). The Malaysian national dental health survey also noted similar trends where the proportion of edentulousness and missing teeth increased sharply after 45 years of age (5).

Dental fatalism and the preference for extractions is a fairly common phenomena among some Malaysian patients (6). It is characterized by a preference to extract a troublesome decayed tooth instead of restoring it, although the latter option is available and has been offered. The missing teeth are later replaced by dentures. In government dental clinics, the three most commonly utilized dental services were fillings, extractions and denture contruction. The relatively high demand for extractions especially, is a disturbing trend $(7,8)$. Furthermore, patients often tend to delay getting the necessary professional attelntion for many months, resulting in a poorer restorative prognosis when the case is finally presented (9). It appears that many subjects had made up their minds about the preferred mode of treatment by the time they come to the dentist.

In order to promote a more positive pattern of dental service utilization behaviour, consumers' attitude and their past utilization behaviour should be assessed. This is because the best predictor of future utilization behaviour is past utilization behaviour (10). The aim of this study is therefore to investigate patients attitude towards the preservation of natural teeth and their past utilization pattern. The objectives are: i) to determine patients own perceptions of whether their natural teeth could be preserved for their lifetime, ii) identify factors which may be associated with this attitude, and iii) establish the relationship between their attitude and past dental service utilization behaviour.

\section{MATERIALS AND METHOD}

The materials consisted of 555 subjects over 15 years of age who attended ten randomly selected government dental clinics in the states of Selangor, Wilayah 
Persekutuan and Negri Sembilan. A timetable was drawn up to visit the clinics during their outpatient's day, totalling thirty nonconsecutive days. The patients were first interviewed by one of three interviewers and then examined clinically by one examiner. The total number seen excluded five subjects who were rejected due to communication and language problems.

Data on the respondents socio-demographic background such as age, sex, ethnic group, education and household income were collected during the interview. The respondents were then asked whether they thought their natural teeth could be preserved for life without the need for full dentures. Alternatively, if they were edentulous, whether they thought this could have been avoided. Their response was classified as either a positive or negative attitude towards preservation of teeth for life.

Respondents were then asked about their last dental visit with regard to the treatment they had sought in the past one year and subsequently were classified into three arbitrary categories. Those whose last dental visit was for an asymptomatic check up were considered as having "good" past utilisation behaviour. If the last dental visit was for restorative or rehabilitative treatment need (eg. carious teeth, bleeding gums or rehabilitation with dentures), the utilization pattern were classified as "fair"; whilst those whose last visit was due to pain or for extractions, were classified as having "poor" utilization behaviour. The variables were analysed using the chi-squared test.

\section{RESULTS}

\section{Composition of sample and dentition status}

Table 1 shows the composition of the sample of utilizers of government dental services by agegroup, sex and ethnic composition. Just under two-thirds of the subjects were females. The ratio of Malays: Chinese: Indians is about 3:1:1. The mean number of missing teeth (MT) is $4.7(\mathrm{SD}+7.1)$. About 33\% ( $n=184)$ had a full natural dentition and the majority of these $(77 \%, n=141)$ were under 24 years old. Only about $3 \%(n=17)$ were totally edentulous, the majority of these $(59 \%, n=10)$ being over 55 years. The frequency of people with a full natural dentition decreased with age, while the opposite was true for the totally edentulous. The correlation between MT and age is strongly positive $(r=0.64, p<0.01)$.

\section{Attitudes towards the preservation of natural teeth for life}

Figure 1 shows the relationship between negative perceptions concerning the preservation of teeth for life and demographic variables. The majority of those examined $(63 \%, n=351)$ had a negative attitude. Negative

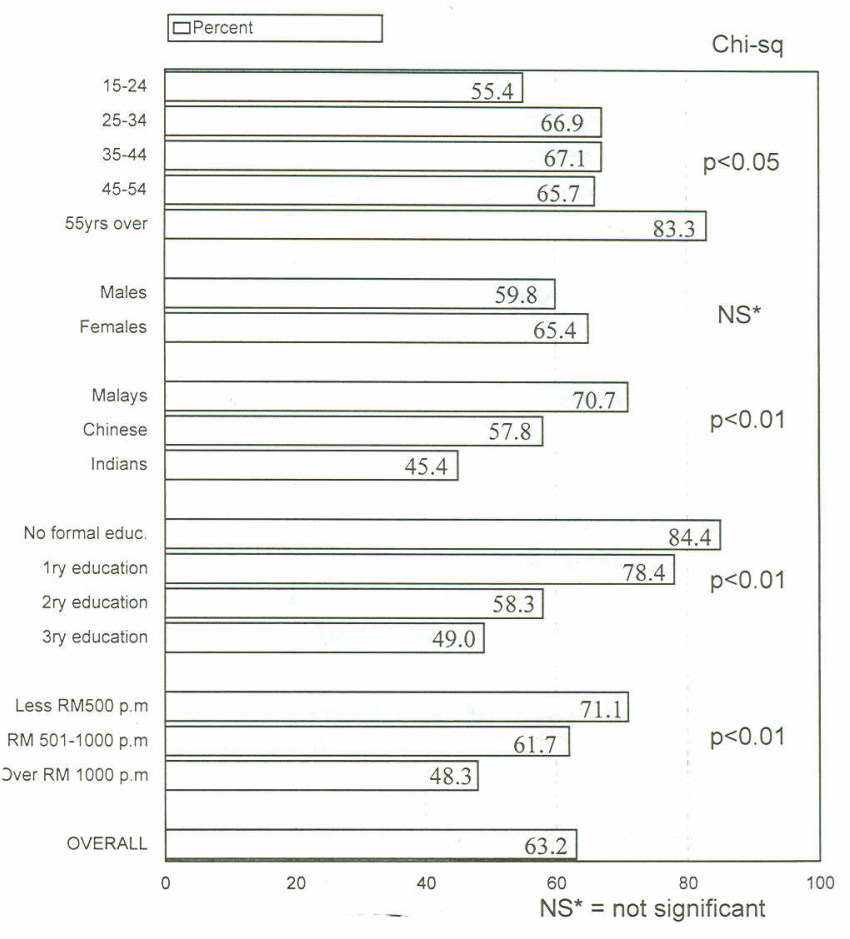

Figure 1. Distribution of subjects with negative perceptions to preservation of teeth for life and demographic variables

attitude was significantly associated with increasing age $(p<0.05)$, the Malay ethnic group $(p<0.01)$, lack of formal education $(p<0.01)$ and lower income $(p<0.01)$. The most educated and highest income group reported the least negative attitudes. The largest proportion with negative opinions among ethnic groups were the Malay respondents $(71 \%, n=239)$, as compared to the Chinese $(58 \%, n=63)$ and least of all, the Indians $(45 \%, n=49)$.

Past dental services utilization behaviour

Figure 2 shows the relationship between past dental service utilization behaviour and demographic variables. About $90 \%(n=497)$ of the overall sample were considered to have a poor utilization behaviour since 
they only sought professional help in response to pain or for extractions. Of all the variables analysed, only education and income were significantly associated with past utilization behaviour $(p<0.01)$. Although the most educated and highest income group had a slightly lower frequency of poor utilization behaviour when compared to the less educated and lower income group, nevertheless the former group's proportion with poor utilization is still very high (around $80 \%$ ).

\section{Attitude towards preservation and and past utilization behaviour}

Table 2 shows the relationship between attitude towards preservation of teeth and past utilization behaviour. It is evident that the majority $(59 \%, \mathrm{n}=327)$ had a poor past utilization pattern as well as a poor attitude towards preservation of natural teeth $(\mathrm{p}<0.01)$. Only $2 \%$ and $8 \%$ had reported a"fair" or "poor" utilization pattern, respectively. In other words, only one in ten subjects had a preventively orientated past utilization behaviour.

Table 2 Relationship between attitude towards the preservation of teeth for a lifetime and past utilization behaviour (Chi sq. $p<0.01$ )

\begin{tabular}{|c|c|c|c|}
\hline \multirow{2}{*}{$\begin{array}{c}\text { Past Utilization } \\
\text { Behaviour }\end{array}$} & \multicolumn{3}{|c|}{ Attitude to Preservation of Teeth } \\
For Life \\
\hline Classification & $\begin{array}{c}\text { Negative } \\
\mathrm{n}(\%)\end{array}$ & $\begin{array}{c}\text { Positive } \\
\mathrm{n}(\%)\end{array}$ & $\begin{array}{c}\text { Total } \\
\mathrm{n}(\%)\end{array}$ \\
\cline { 2 - 4 } Poor & 327 & 171 & $498(90)$ \\
Fair & 8 & 3 & $11(2)$ \\
Good & 17 & 29 & $46(8)$ \\
Total & $352(63 \%)$ & $203(37 \%)$ & $555(100 \%)$ \\
\hline
\end{tabular}

\section{DISCUSSION}

The data must be interpreted with caution because overall, the sample could only reflect the composition of typical utilizers of government dental services. It may not therefore reflect the population as a whole since the public health service in Malaysia is mainly targeted for the less fortunate (14). The higher proportion of young adult utilizers is expected because the Malaysian population structure is relatively young. In other words the sample is therefore not atypical when compared to the general population age structure (11). Furthermore, the impact of caries is most pronounced among young adults.

The national dental survey in 1990 revealed that $7.3 \%$ of adults between the age of 35-44 years old were edentulous, as compared to $17.6 \%$ in the present survey of out-patients (Figure 3) and an estimated 10\% edentulousness in the 1975 national survey of adults in Peninsular Malaysia. This probably indicates that the rate of tooth loss among middle age adults over the 15 year period has not changed very much.

However the overall edentulousness figures in Malaysia are much lower when compared to the $33 \%$ edentulousness rates in the same age group once

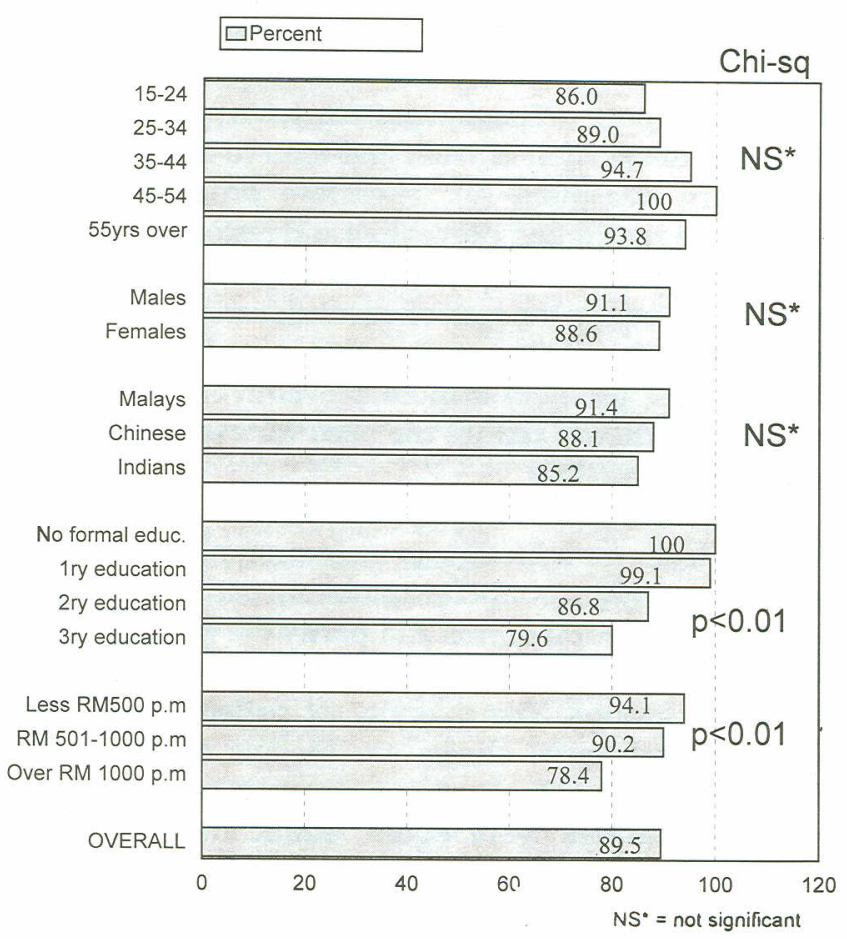

Figure 2. Distribution of subjects with a poor past utilization behaviour and demographic variables

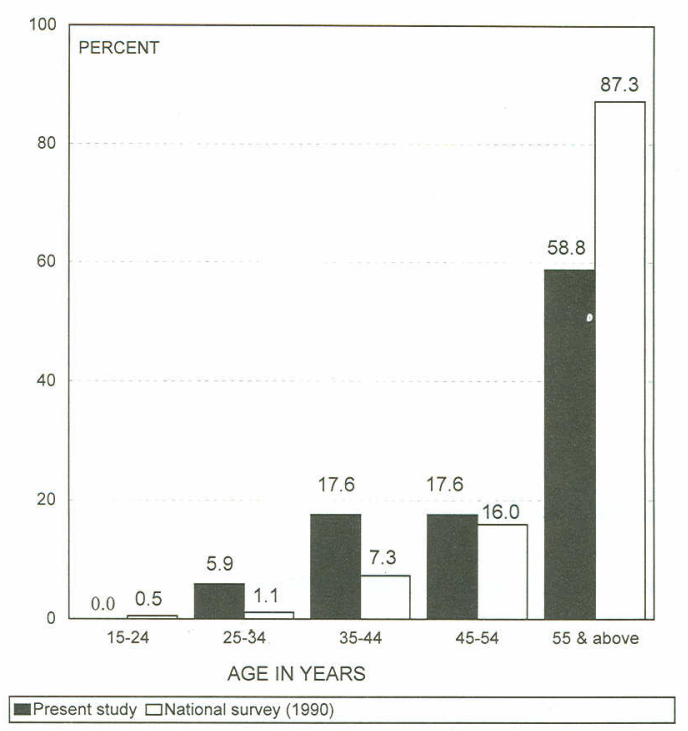

FIG 3. Comparison of edentulousness between present study and the National Adult Dental Health Survey.

experienced by New Zealand in 1978 (3). Therefore the problem of edentulousness at middle age in Malaysia is "relatively" not too serious. Nevertheless, at 35-44 years of age, total loss of all natural teeth is not an acceptable outcome, considering the availability of technology of prevention and restoration.

The implication is that while many teeth may have been saved through expensive restorations at a younger age, they seem to be quickly lost at middle age, probably due to lack of follow-up of treatment and prevention once the subjects leaves the school system. The school dental service delivery system in Malaysia offers both preventive and treatment services to schoolchildren for free without them having to actively seek this service. However once they leave the school system, continuing 
dental care is apparently lost. The majority of adults have a very poor utilization pattern, have poor attitudes toward preservation of teeth and tend to delay getting necessary treatment (9). Therefore programmes to encourage continuing preventive and restorative dental care among adults after they leave the school system is needed to reduce the rate of edentulousness at middle age. It is therefore imperative that dental services for adults must promote a more preventively orientated service in order to reduce the need for extractions and the rate of tooth loss.

The most pessimistic opinion towards retention of natural teeth for a lifetime is most pronounced among the Malay ethnic group. It is probably partly due to the product of cultural norms and partly the fact that most of the Malays account for the lower income and lower educated groups. The majority of patients attending government dental clinics are Malays of the lower income groups (13). Furthermore, in a related study, when all factors were equal, ethnic differences in preference to extractions were not significant (6). Only education and income could account for the variations in preference. Nevertheless, the influence of culture on dental health beliefs and practices among the Malays should be further investigated.

The study also confirms that the majority of utilizers of government dental services do so in response to pain or discomfort. Only about one in ten dental visits by adults may be considered as preventively orientated. The habit of preventive oral health visits is still not an accepted cultural norm. A preventively orientated utilization behaviour among adults is crucial to improve overall health status. The poor pattern of preventive utilization behaviour has two important implications.

First, higher formal education and higher income is significantly associated with better utilization behaviour. Both factors are also important factors influencing socioeconomic development of a community. It follows therefore that long term improvements in oral health, quality of life and proper utilization behaviour can only be sustained.by concomitant improvements in the socioeconomic and physical environment. The needs to fulfill basic needs to survive, often outweighs other considerations such as the health consequences of present actions, including future anticipated benefits of preventive dental visits.

Second, further research concerning utilization behaviour among Malays and the lower socioeconomic group in general, should be made a priority, in order to plan programmes for these target groups as a short term measure. Perceived barriers should be identified and specific programmes including health education and promotion be instituted. The aim of such a programme should be to educate the public that the dentition, with proper care, can be maintained to last for a lifetime. Proper care should include the right attitude towards self care, preservation of natural teeth and preventive utilization behaviour. This will strengthen the stated objective of improving health and providing affordable quality care for the less fortunate groups in Malaysia (14).

In conclusion, the survey found that the majority of Malaysian utilizers of government oral health care services have a rather pessimistic perception of their ability to preserve their own natural teeth for life, have a poor pattern of utilization behaviour and tend to use services mainly for symptomatic reasons. This trend is significantly influenced by their educational and income status.

\section{REFERENCES}

1. Ainamo A, Ainamo J. The dentition is intended to last a lifetime. Int Dent J 1984; $34: 87-92$.

2. Cohen LK. International comparisons in the provision of oral health care. Br Dent J 1980; 149: $347-51$.

3. Barmes DE. A progress report on adult data analysis in the WHO/USPHS International Collaborative Study. Int Dent J1978; 28: 34860.

4. Cohen LK. Implications of findings for dental care across cultures. Int Dent J 1978; 28: 383-6.

5. Dental Division, Min. Of Health. Dental epidemiological survey of adults in Malaysia 1990. Dent. Div. Min. Of Health 1993.

6. Razak IA, Jaafar N, Jallalludin RL, Esa R. Patients preference for exodontia versus preservation in Malaysia. Community Dent Oral Epidemiol 1990; 18: 131-2.

7. Razak IA, Jaafar N. Dental needs, demand and patterns of service utilization in a selected Malaysia urban population. Community Dent Oral Epidemiol 1987; 15: 188-91.

8. Jaafar N, Razak IA. Reasons for seeking dental care in a Malaysian urban adult population: an analysis by sex and ethnic group. Community Dent Oral Epidemiol 1988; 16: 75-8.

9. Jaafar N, Jallalludin RL, Razak IA, Esa R. Investigation of delay in utilization of government dental services in Malaysia. Community Dent Oral Epidemiol 1992; 20: 144-7.

10. Kegeles SS. Why people seek dental care: a test of a conceptual formulation. J Health \& Human Behav 1963; 4: 166-73.

II. Department of Statistic Malaysia. Social Statistic Bulletin. Kuala Lumpur: Government Printer 1986.

12. Dental Division, Min. Of Health. Dental epidemiological survey of adults in Peninsular Malaysia 1975. Dent. Div. Min. Of Health 1977.

13. Latifah R, Nasruddin J, Razak IA, Esa R. Who attends government dental clinics. A socio-economic appraisal of patients. (Abstract) J Dent Res 1990; 69: 1098.

14. Dental Division, Ministry of Health Malaysia. Dental Services . Min. Of Health 1986. 